women 0.86-0.89; men 0.91-0.94), depression (OR's women 4.40-6.82; men 5.25-9.26), BMI (OR's women 1.11-1.16; men 1.10-1.15) and smoking status (OR's $\geq 20$ cigarettes per day women 3.07-7.22; men 2.13-4.54) were associated with fair/poor SRH. No interactions were found between country and grip strength (OR's women 0.95-1.03; men 0.99-1.05) or depression (OR's women 0.63-1.39; men 0.50-1.22) but were found for BMI (OR's women 0.89-0.98; men 0.87-0.97) and smoking (OR's $\geq 20$ cigarettes per day women 0.12-0.34; men $0.20-0.55)$. The interaction between country and BMI reduced when the analysis was restricted to those with a BMI less than 30 (OR's women 0.93-1.07; men 0.90-1.05).

Conclusion Our findings agree with previous research that SRH captures general physical and mental health similarly across countries. We may need more caution comparing SRH across countries when considering other aspects of health. We find that cardiovascular risk has different associations with $\mathrm{SRH}$ in England and Japan, possibly reflecting differences in cultural norms and different stages in their obesity and tobacco epidemics.

\section{P81 FORECASTING TRENDS IN DISABILITY IN ENGLAND AND WALES TO 2030: A MODELLING STUDY}

${ }^{1} \mathrm{M}$ Guzmán-Castillo*, ${ }^{2} \mathrm{~S}$ Ahmadi-Abhari, ${ }^{1,3} \mathrm{P}$ Bandosz, 'S Capewell, ${ }^{2} \mathrm{~A}$ Steptoe, ${ }^{2,4} \mathrm{~A}$ Singh-Manoux, ${ }^{2} \mathrm{M}$ Kivamaki, ${ }^{2} \mathrm{MJ}$ Shipley, ${ }^{2} \mathrm{EJ}$ Brunner, ${ }^{1} \mathrm{M}$ O'Flaherty. ${ }^{1}$ Department of Public Health and Policy, University of Liverpool, Liverpool, UK; ${ }^{2}$ Department Epidemiology and Public Health, University College London, London, UK; ${ }^{3}$ Department of Prevention and Medical Education, Medical University of Gdansk, Gdansk, Poland; ${ }^{4}$ nserm, U1018, Centre for Research in Epidemiology and Population Health, Villejuif, France

\subsection{6/jech-2017-SSMAbstracts.182}

Background Reliably estimating the future burden of disability in our ageing societies is crucial. However, previous forecasts failed to consider the potentially significant impact of trends in disease incidence, both up and down. Our aim is thus to forecast the future disability burden in England and Wales to 2030, while taking into account ongoing trends in CVD and dementia incidence.

Methods We developed and validated the IMPACT-Better Ageing Model. Using evidence-based age-sex- and year-specific transition probabilities, this probabilistic model tracks the England and Wales population aged 35-100 years through ten health states (notably CVD, cognitive impairment, disability, dementia and death). We projected continuing declines in dementia incidence, CVD incidence and mortality rates through to 2030 (based on ELSA analysis and consistent with cohorts elsewhere). We then estimated future disability prevalence, distinguishing four types of disability: CVD-related, dementia-related, CVD and dementia-related and Non-CVD/ Non-dementia disability.

Results England and Wales will continue its transition towards a much older population structure. Between 2015 and 2030, the number of people aged over 65 years will increase by approximately 33.2\% (95\% uncertainty interval 32.2\%-34.1\%) while the very old population (over 85) will increase by some $68.3 \%(63.2 \%-73.0 \%)$

The standardised prevalence of disability among adults aged over 65 years will stay constant at around $21.4 \%(21.2 \%-$ $21.6 \%)$. However, the number of people living with disability will rise by $40.1 \%$ from 2.3 million to 3.2 million.

In 2030, Non-CVD/Non-dementia related disability will account for approximately two-thirds of disabled people aged
65-84, (the absolute numbers increasing in approximately $52 \%$ from 2015). Dementia-related disability will also increase by some 51\% (44.4\%-58.8\%). Conversely, CVD-related disability will decline by approximately 27\% (22.2\%-30.0\%).

Among the very old aged over 85 years, $40 \%$ of the cases of disability in 2030 will be attributable to dementia, representing a 113.4\% (102.6\%-123.6\%) increase since 2015. Non-CVD/Non-dementia disability will rise even more, by approximately 159.2\% (148.4\%\%-169.1\% ). However, CVDrelated disability cases, will increase by just $2.8 \% \quad(-1.4 \%-$ 7.0\%).

Conclusion The number of older people with care needs will expand by approximately one third by 2030, mainly reflecting population ageing rather than higher prevalence rates of disability. This will pose a substantial societal challenge and increase the need for cost-effective long-term care in all its forms: institutional, home-based and informal.

Future research on the potential benefits of effective prevention strategies might therefore concentrate on the shared determinants of these non-communicable diseases.

\section{P82 AN ILLUSTRATION OF THE ANALYTICAL CHALLENGES DUE TO MATHEMATICAL COUPLING IN HEALTH GEOGRAPHY RESEARCH}

${ }^{1,2}{ }^{2}$ Berrie* ${ }^{3}$ PD Norman, ${ }^{1,2} \mathrm{PD}$ Baxter, ${ }^{1,2} \mathrm{MS}$ Gilthorpe. 'Leeds Institute for Data Analytics, University of Leeds, Leeds, UK; ${ }^{2}$ Division of Epidemiology and Biostatistics, University of Leeds, Leeds, UK; ${ }^{3}$ School of Geography, University of Leeds, Leeds, UK

\subsection{6/jech-2017-SSMAbstracts. 183}

Background It has been acknowledged that the use of ratio variables is problematic in regression analysis where ratios comprise common components and are present in both the dependent and independent constituents of the model, e.g. regression analyses of ratio variables with common population denominators. However, such ratio variables are ubiquitous in health research and their resultant mathematical coupling (MC) has not been investigated extensively in relation to studies in health geography, where common population denominators are frequently encountered. It is common, for instance, that area level measures for health outcomes are considered in relation to area levels of mortality and/or indicators of social deprivation, where the common denominator is the area population. Our study seeks to illustrate this issue and examines the implications of this form of $\mathrm{MC}$ from a causal inference perspective.

Methods We examine the impact of MC amongst ratio variables in regression analyses using simulated data based on the correlation structure and distribution of variables derived from the UK census. Specifically, we consider the proportion of limiting long term illness (LLTI) in relation to mortality rates and the Townsend Material Deprivation Score (constructed from percentages of the population of an area that are experiencing pre-defined properties). Simulations are constructed under the null hypothesis, i.e. there is no impact of an area measure of deprivation on the relationship between mortality and proportion of limiting long term illness. A causal framework is introduced utilising directed acyclic graphs (DAGs) to assess variable relationships and to suggest analytical strategies that mitigate any problems arising due to MC.

Results We show that artefactual relationships arise in the regression analyses of composite proportions due to MC: area measures of deprivation appear to influence the relationship 
between LLTI and mortality when no such influence is present in the simulated data. A DAG aids comprehension of this issue from a causal inference perspective and, depending upon the exact nature of the MC present, the DAG can also point to alternative analytical strategies that are discussed.

Conclusion Mathematical coupling of ratio variables has been recognised and reported on in the past, yet its problems remain pervasive. By setting the problem within a causal framework, we provide a means by which the issue might be more readily identified. Furthermore, using DAGs can help direct alternative analytical strategies to remove bias due to MC from future health research.

\section{P83 EXPLAINING THE FALL IN CORONARY MORTALITY IN ARGENTINA BETWEEN 1995 AND 2010: IMPACT MODELLING ANALYSIS}

'J Vicens, ${ }^{1} \mathrm{G}$ Perman, ${ }^{2} \mathrm{P}$ Bandosz, ${ }^{2} \mathrm{M}$ Guzman*, ${ }^{3} \mathrm{D}$ Ferrante, ${ }^{1} \mathrm{H}$ Schargrodsky, ${ }^{1} \mathrm{~V}$ Aliperti, P Pramparo, ${ }^{2} \mathrm{M}$ O'Flaherty, ${ }^{1} \mathrm{~S}$ Figar. ${ }^{1}$ Department of Internal Medicine and Cardiology, Hospital Italiano de Buenos Aires, Buenos Aires, Argentina; ${ }^{P}$ Public Health and Policy, University of Liverpool, Liverpool, UK; ${ }^{3}$ Ministry of Health, City of Buenos Aires Government, Buenos Aires, Argentina

\subsection{6/jech-2017-SSMAbstracts.184}

Background Coronary heart disease (CHD) mortality has declined substantially in Argentina in the last decades. However, the contributory factors remain unclear because major CHD risk factor levels have paradoxically increased, notably physical inactivity, obesity and diabetes.

our objectiv is to quantify the contributions of prevention and treatment to the coronary heart disease mortality trends in Argentina between 1995 and 2010.

Methods We used the previously validated IMPACT model to analyse mortality trends between 1995 and 2010 in the Argentinean population aged 25 years and over. This model integrates data on changes in population size, CHD mortality, risk factors, and uptake of evidence-based cardiac treatments. Main data sources included official vital statistics, national health surveys, and published papers in the scientific literature. If none of these were available, we used expert judgement. Relative risks and regression coefficients came from the published meta-analyses. The difference between observed and expected CHD deaths in 2010 was then partitioned among treatment benefits and risk factor changes. We also performed probabilistic sensitivity analyses to quantify the potential effects of parameter uncertainty.

Results From 1995 to 2010, age-adjusted CHD mortality rates in Argentina fell by 24.1\%, resulting in 8500 fewer CHD deaths in 2010 had mortality rates remained unchanged. Improvements in medical and surgical treatments were associated with 53.8\% (95\% uncertainty interval, 46.2\%-61.8\%) of the total mortality decrease. Major contributions came from heart failure therapies (15.1\%), antihypertensive medication $(11.9 \%)$, and secondary prevention following acute myocardial infraction (8.2\%).

Risk factor changes accounted for 40.4\% (9.5\% to $65.6 \%$ ) of the mortality fall. Decreases in systolic blood pressure accounted for $34.6 \%$; in cholesterol levels, 19.9\%; and in smoking prevalence, $6.7 \%$. However, rises in diabetes, BMI and physical inactivity negated some of these benefits, potentially increasing mortality by $9.4 \%, 6.4 \%$ and $5.0 \%$, respectively. Approximately, $6 \%$ of the deaths prevented or postponed could not be explained in our model.
Results Approximately 54\% of the CHD mortality fall in Argentina between 1995 and 2010 was attributable to increases in evidence-based medical treatments, and $40 \%$ to falls in population risk factors. However, the substantial contributions from falls in blood pressure, cholesterol and smoking were offset by adverse trends in diabetes, obesity and physical inactivity. Our results highlight the potential for further improvements in cardiovascular

\section{P84 DEFINING A PRIMARY COMPOSITE OUTCOME FROM HOSPITAL EPISODE STATISTICS DATA TO TEST THE BENEFIT OF CARDIAC MAGNETIC RESONANCE (CMR) IMAGING AFTER PRIMARY PERCUTANEOUS CORONARY INTERVENTION (PPCl)}

${ }^{1} \mathrm{M}$ Pufulete*, ${ }^{1} \mathrm{~J}$ Harris, ${ }^{2} \mathrm{~S}$ Dorman, ${ }^{1} \mathrm{R}$ Brierley, ${ }^{1} \mathrm{~B}$ Reeves. ${ }^{1}$ Clinical Trials and Evaluation Unit, University of Bristol, UK; ${ }^{2}$ Bristol Heart Institute, University Hospitals Bristol NHS Foundation Trust, UK

\subsection{6/jech-2017-SSMAbstracts. 185}

Background We researched the feasibility of using routinely collected data to establish a registry to document the use of cardiac magnetic resonance imaging (CMR) in patients with suspected heart attack who activate the primary percutaneous coronary intervention (PPCI) pathway. To compare outcomes between patients having CMR or not, we aimed to define a primary composite outcome, representing clinically important changes in management resulting from CMR, using data from Hospital Episode Statistics (HES) or the Patient Episode Database Wales (PEDW). Clinically important changes in management, and patient subgroups to which these related, were defined by formal consensus.

Methods Patients at four UK sites were prospectively consented. We assembled a database by linking hospital-collected data about the index PPCI admission (demography, clinical, biochemistry and imaging) and CMR 'exposure' within 10 weeks with HES/PEDW data for the following 12 months. An experienced medical coder identified ICD10 diagnostic codes and OPCS procedure codes that the pre-defined clinically important changes in management achieved by CMR were hypothesised to influence. Episodes in HES/PEDW inpatient, outpatient and accident and emergency datasets with these codes were identified. This process was applied to key patient subgroups: i) PPCI; ii) multivessel coronary disease; iii) out-ofhospital cardiac arrest; iv) coronary angiogram showing unobstructed arteries.

Results Of the 1670 patients consented, 1476 (88\%) had PPCI; 682 (41\%) had multivessel disease, and 194 (12\%) had unobstructed arteries. 189 (11\%) had CMR within 10 weeks of the index admission. 1612 (97\%) had their index event identified in HES/PEDW and 985 of these (61\%) had a full year of follow-up available. Focusing on important changes by patient subgroup avoids creating a composite outcome based on diverse changes. Frequencies of relevant health episodes will be reported for patient subgroups and by CMR exposure. Comparing frequencies is straightforward but identifying better targeting of care to patients' needs without a change in frequency, e.g. due to better diagnostic accuracy of CMR, is complex.

Discussion Clinical events relating to important changes in management resulting from CMR can be identified in HES but CMR may not alter the frequency of these events. This case study demonstrates the promise of using routinely 